\section{Shift anticipated in DNA microarray market}

$R^{\mathrm{s}}$ ecent activity in the microarray arena reaffirms Affymetrix's (Santa Clara, CA) stronghold on the high-density DNA array market. However, although the demand for microarrays is expected to double in the next five years, much of this is likely to be for lowdensity platforms, and significant opportunities still exist for the $\sim 25$ firms that are currently jockeying for position in the broader market.

At present, about $75 \%$ of microarray research involves broad gene expression profiling using high-density arrays to screen cellular messenger RNA samples across as many polynucleotide probes as possible. The market for pre-arrayed DNA slides is worth about $\$ 300$ million, and Affymetrix currently holds about $80 \%$ of it, according to Ken Smith of business strategy and marketing consultants, Front Line Consulting (Foster City, CA).

Affymetrix's success is due to its photolithographic technology, which is similar to that used by the semiconductor industry and enables Affymetrix to make the highest density DNA microarrays available: Affymetrix can currently build over 400,000 features (multiple copies of a probe) on a $1.64 \mathrm{~cm}^{2}$ chip, but a chip bearing over 40 million features is theoretically possible. The maximum density achievable by other commercial technologies available-ink-jet printing (Agilent) and deposition (Motorola, among others) - is limited by the volume of liquid that must be deposited to transfer probes onto a slide. Thomas Flaten of equity research group Dain Rauscher Wessels (Minneapolis, MN) believes the closest competitor will be Agilent, which plans to develop microarrays of about 100,000 features per $1.64 \mathrm{~cm}^{2}$ (its current $19.35 \mathrm{~cm}^{2}$ chip holds a total of 27,000 probes), but that Affymetrix is the only firm that could conceivably place the entire human genome on a single chip. "Given that our understanding of the genomic basis for disease continues to be an evolving field," he says, "it seems obvious that people would want to know as much as possible as quickly as possible."

Ted Love, CEO of Hyseq (Sunnyvale, CA), says "There's nobody that can block Affymetrix from doing what they do [by invalidating its photolithography patents in the courtroom]." Hyseq, which was developing a DNA microarray for gene sequencing, recently restructured to become Hyseq Pharmaceuticals, but will stay in the microarray business through $\mathrm{N}-\mathrm{Mer}$, its joint venture with Affymetrix formed through a patent litigation settlement (Nat. Biotechnol. 19, 193; 2001). N-Mer aims to develop a universal high-density chip for gene sequencing.

Other companies have also recently bowed out of the sector. In October, Incyte Genomics (Palo Alto, CA), which was trying to market high-density arrays from its complementary DNA libraries, restructured itself as a virtual pharmaceutical company, citing "increasing competition and margin erosion" as a reason to discontinue with its microarray ventures (Nat. Biotechnol. 19, 1092; 2001). Around the same time, Corning (Corning, NY) axed its microarray efforts, which included two marketed gene chips and a 10,000-spot human array in development. Scott Greenstone, research analyst with Thomas Weisel Partners (New York), says that Corning's science was sound, but market conditions made further investment outside of its core business of fiber optics unwise, especially as Corning was a late entry to the field. Consequently, few companies are left to challenge Affymetrix in the high-density market.

However, while the market for DNA microarrays will probably reach $\$ 681$ million by 2006, according to Front Line's November 19 report DNA Microarrays: A Strategic Market Analysis, much of this growth is expected to come from an increased demand for low-density micorrays. Smith explains that once a relatively small number of genes has been associated with a disease (the goal of current research using high-density arrays), lowdensity arrays would be used to screen those small number of genes across many patients. However, associating genes with disease is at a bottleneck, and many more population genetics studies and improved informatics algorithms are demand for lowdensity microarrays will soar-something that could take five years, says Smith.

In the meantime, several companies, including Affymetrix, are positioning themselves in different parts of the broadening market (see Table). Agilent, for example, while marketing high-density microarrays, also custom-designs needed before lower-density arrays for researchers. And CombiMatrix plans to join the custom-array business with its electrochemical technology that builds probes directly on the chip.

Others, such as GeneMachines (San Carlos, CA), Apogent (Portsmouth, NH), and Affymetrix are leaders in selling arrayers, machines that researchers can use to make their own microarrays. The companies hold $17 \%, 21 \%$, and $35 \%$, respectively, of the $\$ 150$ million market, which, according to Front Line, will grow $44 \%$ per year to $\$ 887$ million in 2006.

Still others are focusing on scanners and image analysis software, which are essential to read microarrays and analyze data-an area Smith believes will grow $44 \%$ per year to about $\$ 1.7$ billion in 2006. Axon (Union City, $\mathrm{CA}$ ) and PerkinElmer (Wellesley, MA) have a strong position in this area.

Meanwhile, several companies have switched from making DNA microarrays for gene expression profiling to using them in SNP-based diagnostics and pharmacogenomics. In May, Nanogen (San Diego, CA) refocused its microfluidics technology from making devices for gene expression profiling to the development of diagnostics. And cash-rich Motorola, which is currently selling a P450 SNP chip for pharmacogenetic profiling, also hopes to move into the diagnostics market.

Most analysts say it's unlikely all the firms currently vying for positions will survive, even though the combined market-of arrayers, low- and high-density chips, hardware, and software-is expected to grow over $30 \%$ per year to $\$ 3.6-4.5$ billion in the next five years. The most likey winners, says Flaten, are those firms, such as Genomic Solutions (Ann Arbor, $\mathrm{MI}$ ), that can provide the whole gamut.

Aaron Bouchie, New York

\begin{tabular}{lll}
\multicolumn{2}{l}{ Selected DNA microarray companies and their products } \\
Scanners & Arrayers & Pre-spotted slides \\
Applied Precision & Apogent & Azign BioScience \\
Axon Instruments & Gene Machines & Clontech \\
Alpha Innotech & TeleChem & Mergen \\
Gene Focus & V\&P Scientific & Origene \\
& Genetix & Phase 1 Molecular \\
& Protogene & Toxicology \\
Agilent & Agilent & \\
Motorola & Motorola & \\
Hitachi & Hitachi & \\
Intelligent & Intelligent & \\
Automation Systems & Automation Systems & \\
RoboDesign & RoboDesign & \\
Virtek Vision & Virtek Vision & \\
& Takara Shuzo & Takara Shuzo \\
Affymetrix & Affymetrix & Affymetrix \\
Amersham Pharmacia & Amersham Pharmacia & Amersham Pharmacia \\
Biotech & Biotech & Biotech \\
Genomic Solutions & Genomic Solutions & Genomic Solutions \\
MWG & MWG & MWG \\
PerkinElmer & PerkinElmer & PerkinElmer \\
\hline
\end{tabular}

Source: company websites 